\title{
Optical Flip-Flop Operation using an AR-coated Distributed Feedback Laser Diode
}

\author{
K. Huybrechts, W. D’Oosterlinck, G. Morthier, R. Baets \\ Department of Information Technology, Ghent University - IMEC, Sint-Pietersnieuwstraat 41, B-9000 Gent, Belgium \\ E-mail: koen.huybrechts@intec.ugent.be
}

\begin{abstract}
A new concept for all-optical flip-flops is introduced using a single DFB laser diode. When injecting external light into the laser, two stable states can be obtained. We show numerically that optical pulses allow switching.

(C)2007 Optical Society of America

OCIS codes: (230.1150) All-optical devices; (060.4510) Optical communications;
\end{abstract}

\section{Introduction}

Packet or burst switched optical networks are gaining a lot of interest due to the increasing demand for faster network traffic [1]. All-optical flip-flops are one of the key building blocks to achieve these optical networks. Several concepts for all-optical flip-flops have been proposed [2-4], but they often suffer from disadvantages such as difficult fabrication (due to a difficult passive active integration), slow switching, high switching energies, requiring tight wavelength control, ...

In this paper we show numerically that optical bistability can be obtained in an AR-coated DFB laser diode which is biased above threshold and in which external light is injected. This bistability is observed in the lasing light as well as in the amplification of the external light. This is illustrated in Figure 1 for a $\lambda / 4$-shifted, AR-coated DFB laser with length $1 \mathrm{~mm}$ and $\kappa \mathrm{L}=0.6$. There are no strict limitations on the wavelength of the injected light (except that it should not be too close to the lasing wavelength) which makes the device suitable for broadband operation. We will use a DFB laser working at $1.57 \mu \mathrm{m}$ and use a wavelength of $1.56 \mu \mathrm{m}$ for the external light. We also show that short optical pulses can be used to switch between the two stable states making all-optical flip-flop operation possible.

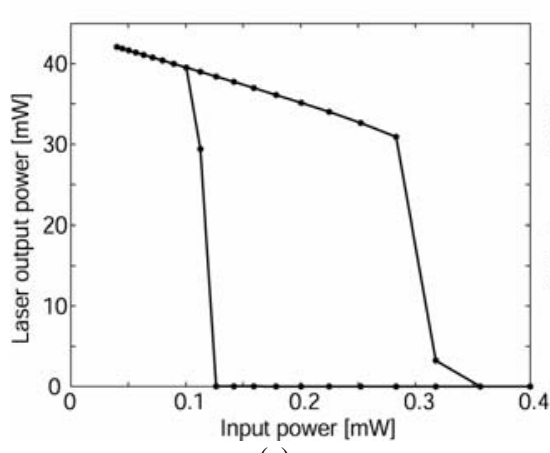

(a)



(b)

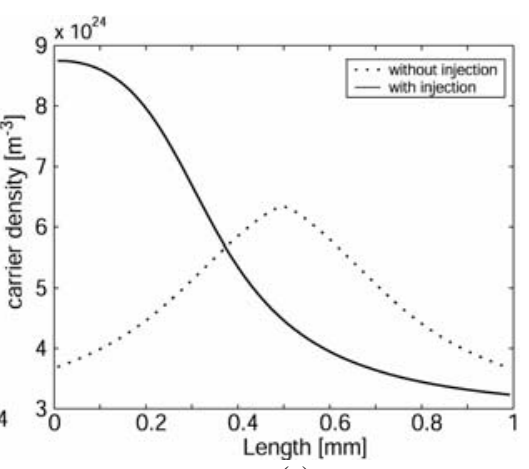

(c)

Figure 1: (a) lasing power vs. injected power for a DFB laser of length $1 \mathrm{~mm}, \kappa \mathrm{L}=0.6$ and $\mathrm{I}_{\text {bias }}=260 \mathrm{~mA}$; (b) amplification of the injected power; (c) the longitudinal distribution of the carrier density for the on-state and the off-state.

\section{Operation Principle}

The bistability arises from the strong influence of spatial hole burning in DFB laser diodes. Under the injection of a CW beam into the laser diode, there exist two stable states: one in which the laser diode is lasing, with low amplification of the injected beam (due to the gain clamping) and another one in which the laser diode is switched off, with high amplification of the injected beam. In the last case, the high amplification causes a strong nonuniformity of the carrier density (Fig. 1c). It is well-known that this spatial hole burning may increase the threshold of a DFB laser diode, ultimately causing the laser to switch off even though the average gain is higher.

One can switch from the lasing state to the non-lasing state by injecting a short and strong optical pulse on one side of the device. Switching from the non-lasing state to the lasing state can then be done by restoring the spatial uniformity by injecting a strong and short optical pulse from the opposite direction. 


\section{Static behavior}

The bistability depends on the parameters of the DFB laser. We have investigated the influence of a.o. the bias current and the normalized coupling coefficient $\kappa L$ for $\lambda / 4$-shifted DFB lasers. Figure 2a shows the influence of the bias current and Figure $2 b$ the influence of $\kappa \mathrm{L}$. The bistable domain gets smaller when decreasing the bias current due to the fact that the laser switches off sooner in the low gain branch. The hysteresis curve also narrows for decreasing $\kappa L$-value as can be seen in Figure $2 b$. This can be explained by the increase of the threshold gain with decreasing $\kappa L$-value. As a result, the amplification of the injected light in the low or clamped gain branch is higher for lower $\kappa \mathrm{L}$ and thus the laser switches off sooner in the clamped gain branch for lower $\kappa \mathrm{L}$.



(a)

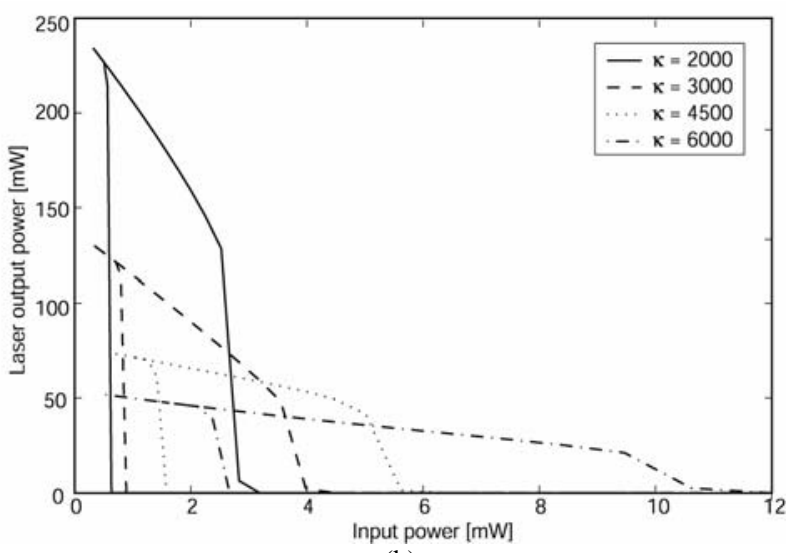

(b)

Figure 2: a) Influence of bias current on hysteresis characteristics for a DFB laser of length $600 \mu \mathrm{m}, \kappa=3000 / \mathrm{m}$ and $\mathrm{I}_{\mathrm{th}}=26.5 \mathrm{~mA}$. b) Influence of coupling coefficient for a laser with length $300 \mu \mathrm{m}$ and $\mathrm{I}_{\text {bias }}=8 \mathrm{I}_{\mathrm{th}}$.

\section{Dynamic behavior}

To study the dynamic properties of the flip-flops, CW light is injected on one side of the laser cavity together with the reset pulses. The set-pulses are injected at the other side of the flip-flop to restore the carrier density uniformity. Our first simulations give switching times of about $300 \mathrm{ps}$, switching energies of $160 \mathrm{fJ}$ and a contrast ratio of $25 \mathrm{~dB}$.

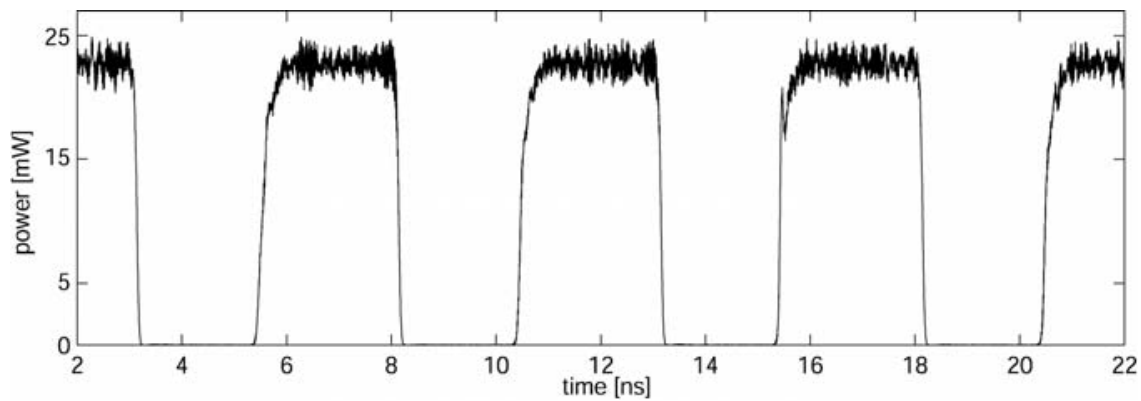

Figure 3: Illustration of the switching properties for the use as a flip-flop $\left(\kappa \mathrm{L}=0.9, \mathrm{~L}=600 \mu \mathrm{m}\right.$ and $\mathrm{I}_{\text {bias }}=200 \mathrm{~mA}$ and CW-power is $0.52 \mathrm{~mW}$ )

\section{Conclusion}

We have shown that an all-optical flip-flop can be obtained using an AR-coated DFB laser with injection of CW light. The shape of the bistability curve depends on the laser parameters and on the bias current.

\section{References}

[1] H.J.S. Dorren, M.T. Hill, Y. Liu, N. Calabretta, A. Srivatsa, F. M. Huijskens, H. de Waardt, G. D. Khoe, "Optical Packet Switching and Buffering by Using All-Optical Signal Processing Methods”, Journal of Lightwave Technology, vol. 21, Jan. 2003, pp. 2-12.

[2] R. Clavero, F. Ramos, J.M. Martinez, and J. Marti. “All-optical flip-flop based on a single SOA-MZI”, IEEE Photonics Technology Letters, Vol. 17(4):843-845, April 2005.

[3] M. T. Hill, H. de Waardt, G.D. Khoe, and H.J.S. Dorren, “All-optical flip-flop based on a coupled laser diodes”, IEEE J. Quantum Electronics, 37(3):405-413, March 2001.

[4] M. Takenaka and Y. Nakano, "Realization of all-optical flip-flop using directionally coupled bistable laser diode", IEEE Photonics Technology Letters, 16(1):45-47, January 2004. 